\title{
Effectiveness of pertussis vaccination and duration of immunity
}

\author{
Kevin L. Schwartz MD, Jeffrey C. Kwong MD, Shelley L. Deeks MD, Michael A. Campitelli MPH, \\ Frances B. Jamieson MD, Alex Marchand-Austin MSc, Therese A. Stukel PhD, Laura Rosella PhD, \\ Nick Daneman MD, Shelly Bolotin PhD, Steven J. Drews PhD, Heather Rilkoff MPH, \\ Natasha S. Crowcroft MD(Cantab)
}

See also www.cmaj.ca/lookup/doi/10.1503/cmaj.161048

\begin{abstract}
- Abstract
Background: A resurgence of pertussis cases among both vaccinated and unvaccinated people raises questions about vaccine effectiveness over time. Our objective was to study the effectiveness of the pertussis vaccine and characterize the effect of waning immunity and whole-cell vaccine priming.
\end{abstract}

Methods: We used the test-negative design, nested case-control study with test-negative individuals as controls. We constructed multivariable logistic regression models to estimate odds ratios (ORs). Vaccine effectiveness was calculated as $(1-$ OR $) \times 100$. We assessed waning immunity by calculating the odds of developing pertussis per year since last vaccination and evaluated the relative effectiveness of priming with acellular versus whole-cell vaccine.

Results: Between Dec. 7, 2009, and Mar. 31, 2013, data on 5867 individuals (486 testpositive cases and 5381 test-negative con-

$\mathrm{W}$ hooping cough, or pertussis, is a highly contagious respiratory infection that has been inadequately controlled compared with other vaccine-preventable diseases. The incidence of pertussis in Canada decreased from 156 cases per 100000 population during the prevaccination era to a historic low of 2.0 per 100000 in 2011, increased to 13.9 in 2012, and then decreased to 3.6 in $2013 .{ }^{1}$

Ontario, Canada's most populous province with a population of 13.5 million in 2013, experienced a localized outbreak in 2012. This outbreak started in a largely unvaccinated religious community and disproportionately affected infants, but then spread to the general population and mostly involved adolescents. ${ }^{2}$ Consequently, the outbreak raised questions about product-specific vaccine effectiveness and waning immunity. trols) were available for analysis. Adjusted vaccine effectiveness was $80 \%$ (95\% confidence interval $[\mathrm{Cl}] 71 \%$ to $86 \%$ ) at $15-364$ days, $84 \%(95 \% \mathrm{Cl} 77 \%$ to $89 \%)$ at $1-3$ years, $62 \%(95 \% \mathrm{Cl} 42 \%$ to $75 \%)$ at $4-7$ years and $41 \%(95 \% \mathrm{Cl} 0 \%$ to $66 \%)$ at 8 or more years since last vaccination. We observed waning immunity with the acellular vaccine, with an adjusted OR for pertussis infection of 1.27 $(95 \% \mathrm{Cl} 1.20$ to 1.34$)$ per year since last vaccination. Acellular, versus whole-cell, vaccine priming was associated with an increased odds of pertussis (adjusted OR 2.15, 95\% Cl 1.30 to 3.57 ).

Interpretation: We observed high early effectiveness of the pertussis vaccine that rapidly declined as time since last vaccination surpassed 4 years, particularly with acellular vaccine priming. Considering whole-cell vaccine priming and/or boosters in pregnancy to optimize pertussis control may be prudent.

Pertussis vaccines have been available in Ontario for more than 70 years. In 1997, owing to concerns about safety and effectiveness, acellular pertussis vaccine replaced the whole-cell product that had been in use since $1984 . .^{3-5}$ In Ontario, only the 5-component acellular vaccine (containing pertussis toxoid, filamentous hemagglutinin, pertactin, and fimbriae types 2 and 3 ) has been available for infants and toddlers. Vaccination against pertussis is recommended at 2 , 4, 6 and 18 months, and at 4-6 years. In 2003, an adolescent dose at 14-16 years was introduced, and in 2011, a program was started for singledose adult vaccination against pertussis. ${ }^{6}$

Other jurisdictions in Canada, the United States and Australia have reported lower effectiveness with the acellular product and rapidly waning immunity. ${ }^{7-11}$ Canada has a unique history of using
Competing interests: None declared.

This article has been peer reviewed.

Accepted: May 30, 2016 Online: Sept. 26, 2016

Correspondence to: Kevin Schwartz, kevin. schwartz@oahpp.ca

CMAJ 2016. DOI:10.1503/ cmaj.160193 
a whole-cell vaccine with lower effectiveness, and also has different secular trends in pertussis incidence and vaccination coverage, necessitating local evaluation of the effectiveness of the pertussis vaccine to inform vaccination policy. Our objective was to study the effectiveness of the pertussis vaccine in Ontario while characterizing the effect of waning immunity and whole-cell vaccine priming.

\section{Methods}

\section{Study population, setting and design}

We linked Bordetella pertussis polymerase chain reaction (PCR) test results from Public Health Ontario Laboratory Services to population-based provincial health administrative data (linkage success rate $96 \%$ ). To assess the effectiveness of the pertussis vaccine, we used the test-negative design, a type of nested case-control study often used to assess the effectiveness of the influenza vaccine because it generates unbiased estimates comparable to those from randomized controlled trials (RCTs). ${ }^{12-14}$

The study population comprised Ontario residents born between Apr. 1, 1992, and Jan. 1, 2013. We excluded records with invalid postal codes, children younger than 3 months and individuals who were registered for coverage in Ontario's universal health care system at 6 months of age or older to account for those who may have received their primary vaccination series elsewhere. We excluded vaccinations given within 14 days of testing owing to insufficient time to mount an adequate immunologic response.

\section{Data sources and definitions}

\section{Laboratory data}

Public Health Ontario's laboratory database contains results for more than 95\% of Ontario's diagnostic testing for pertussis; testing is available to all Ontario physicians. The laboratory methods have been previously described..$^{15-17}$ Briefly, until May 28, 2012, primers targeting the insertion sequence IS481 were used to detect $B$. pertussis. Following this date, primers targeting a 50-base pair segment of the $r e c A$ gene were also included to distinguish $B$. pertussis from Bordetella holmesii. Evaluation of the new assay showed that less than $1 \%$ of previously tested pertussis-positive specimens were $B$. holmesii. ${ }^{17}$ Polymerase chain reaction tests were considered positive for pertussis at cycle threshold values of 35 or less, and indeterminate at 36-40. Specimens with no amplification signal were considered negative. These values were constant throughout the study period.
Data were available from Dec. 7, 2009, to Mar. 31, 2013. We defined cases as individuals who tested positive for $B$. pertussis and controls as those who tested negative. Indeterminate results were excluded from the primary analysis. Patients who received multiple tests within a 90-day period were considered to have tested positive if they had at least 1 positive result, indeterminate if they received at least 1 indeterminate and no positive results, and negative if they received only negative results. No cases had more than 1 positive test more than 90 days apart.

\section{Pertussis vaccination}

The database of the Ontario Health Insurance Plan (OHIP) contains physician-billing information on virtually all Ontario residents starting Jan. 1, 1992, except for new residents within their first 3 months of residency. ${ }^{18}$

We classified vaccination status as up to date for age, partially vaccinated or unvaccinated. The number of age-appropriate doses is 1 dose at 3 months, 2 doses at 5 months, 3 doses at 7 months, 4 doses at 19 months, 5 doses at 7 years and 6 doses at 17 years, based on Ontario's immunization schedule. ${ }^{6}$ We defined a priming series as the first 3 doses of vaccine received. Vaccines in Ontario are purchased centrally for the entire province. The Connaught whole-cell vaccine was available between 1992 and 1996, and only acellular vaccine in 1998 and later. Vaccines administered in 1997 were used in a sensitivity analysis because of uncertainty regarding the overlap of products used that year. Ontario's immunization codes have been previously validated in infants. ${ }^{19}$ We performed a further validation analysis of pertussis codes for this study (Appendix 1, available at www.cmaj.ca/ lookup/suppl/doi:10.1503/cmaj.160193/-/DC1).

\section{Covariates}

We used Ontario's Registered Persons Database, which contains demographic information on all individuals with a valid Ontario health card, ${ }^{20}$ to obtain age, sex, socioeconomic status (approximated by neighbourhood income quintile $)^{21}$ and rural residence (community size $<10000$ ). We used the Canadian Institute for Health Information (CIHI) Discharge Abstract Database to identify chronic conditions. ${ }^{22}$ Health care use within the previous year (including physician visits, emergency department use and hospital admissions) was enumerated using OHIP, the CIHI National Ambulatory Care Reporting System and the Discharge Abstract Database, respectively. These data sets were linked using unique encoded identifiers and analyzed at the Institute for Clinical Evaluative Sciences. 


\section{Statistical analysis}

The proportion of pertussis cases by time since last vaccination was evaluated using the Cochran-Armitage trend test. We used multivariable logistic regression models to estimate crude and adjusted odds ratios (ORs) for pertussis infection, assessing participants with up-to-date or partial vaccination compared with unvaccinated participants. We stratified the data by time since last vaccination: 15-364 days, 1-3 years, 4-7 years, and 8 or more years since last vaccination. These periods were selected in relation to age-based vaccination recommendations and based on expected waning of immunity from previous studies. ${ }^{10,23}$ The variables age, sex, any comorbidity, ${ }^{22}$ income quintile, rural residence, ambulatory, emergency and inpatient health care use in the past 12 months were selected a priori for inclusion in the multivariable models. We calculated vaccine effectiveness as $(1-\mathrm{OR}) \times 100 .^{24}$

Waning immunity of the acellular pertussis vaccine was further assessed in those born in 1997 or later by estimating the odds of pertussis for each year following the last vaccine dose received, adjusted for the same covariates above, among those with up-to-date vaccination, those with either up-to-date or partial vaccination, and those who had received 5 or more previous doses of pertussis vaccine (i.e., older individuals who should have received the preschool diphtheria and tetanus toxoids and acellular pertussis [DTaP] or tetanus toxoid, reduced diphtheria toxoid, and acellular pertussis [Tdap] vaccine).

We evaluated the relative impact of priming with acellular versus whole-cell vaccine in those aged 10 years and older who had received 5 or more pertussis vaccinations. We constructed multivariable logistic regression models to estimate the odds of pertussis if the priming series used acellular vaccine only compared with whole-cell vaccine only, and compared with 1 or more doses of whole-cell vaccine.

We used the PROC LOGISTIC procedure (SAS 9.3) for all analyses. Type 1 error was set at $5 \%(p<0.05)$. We evaluated all variables for multicollinearity through the variance inflation factor. Model fit was evaluated with the Hosmer-Lemeshow test.

\section{Sensitivity analysis}

Because of the potential nondifferential misclassification bias of vaccination status from physician billing claims, we performed a quantitative sensitivity analysis that examines the impact of the known sensitivity and specificity of the exposure variable on the estimate of vaccine effectiveness. ${ }^{25}$ Details are in Appendix 2, available at www.cmaj.ca/lookup/suppl/doi:10.1503/
cmaj.160193/-/DC1. We evaluated the impact of an incomplete primary series by excluding infants younger than 7 months. We also analyzed indeterminate pertussis results, separating time periods (2009-2011 v. 2012-2013) and excluding cases from a localized outbreak.

\section{Ethics approval}

We received ethics approval from Public Health Ontario, Sunnybrook Health Sciences Centre and the University of Toronto.

\section{Results}

We linked 7560 individuals born after Apr. 1, 1992, with a pertussis PCR test result between Dec. 7, 2009, and Mar. 31, 2013. We excluded 1112 infants younger than 3 months, 361 people who registered for Ontario health insurance at age 6 months or older, and 220 people with indeterminate results. The final study population included 5867 participants (486 pertussis-positive cases and 5381 pertussis-negative controls).

Cases were older than controls by a mean of 2.7 years (Table 1 ). Cases were more likely to reside in rural areas and to have been tested in 2012. Controls had higher rates of comorbidities and had accessed more health care in the previous 12 months.

Of the 486 cases, 193 (39.7\%) had up-todate vaccination against pertussis according to our definition, $134(27.6 \%)$ were partially vaccinated and 159 (32.7\%) were unvaccinated. Among the 5381 controls, 3384 (62.9\%) were up to date, $1212(22.5 \%)$ were partially vaccinated and 785 (14.6\%) were unvaccinated. Of the participants, $120(24.7 \%)$ cases and 1121 $(20.8 \%)$ controls had received 5 or more doses.

Unadjusted vaccine effectiveness declined over time by about $10 \%$ per year since last vaccination in those with up-to-date or partial vaccination (Figure 1). Correspondingly, the proportion of positive cases increased by about $2 \%$ each year since last vaccination $(p<0.001$, by the Cochran-Armitage trend test).

Compared with unvaccinated participants, the adjusted estimates of vaccine effectiveness for participants who had up-to-date vaccination, stratified by time since last vaccination, were $80 \%$ (95\% CI $71 \%$ to $86 \%$ ) at $15-364$ days, $84 \%$ (95\% CI $77 \%$ to $89 \%)$ at $1-3$ years, $62 \%(95 \%$ CI $42 \%$ to $75 \%)$ at $4-7$ years and $41 \%(95 \%$ CI $0 \%$ to $66 \%$ ) at 8 or more years since last vaccination (Table 2). The trend was similar when we compared the partially vaccinated group with the unvaccinated group.

Individuals who received only an acellular vaccine had significant waning of immunity. 
For each year that elapsed from acellular pertussis vaccination, there was a $27 \%$ increased odds of testing positive for pertussis among those with up-to-date vaccination for their age (adjusted OR 1.27, 95\% CI 1.20 to 1.34). The sensitivity analyses had similar results, with an adjusted OR of 1.21 (95\% CI 1.17 to 1.26 ) for

\begin{tabular}{|c|c|c|}
\hline \multirow[b]{2}{*}{ Characteristic } & \multicolumn{2}{|c|}{ No. $(\%)^{*}$} \\
\hline & $\begin{array}{c}\text { Pertussis-positive } \\
\text { cases } \\
n=486\end{array}$ & $\begin{array}{c}\text { Pertussis-negative } \\
\text { controls } \\
n=5381\end{array}$ \\
\hline Age, yr, mean $\pm S D$ & $7.7 \pm 5.7$ & $5.0 \pm 5.0$ \\
\hline \multicolumn{3}{|l|}{ Age } \\
\hline $3-11 \mathrm{mo}$ & $80(16.5)$ & $1268(23.6)$ \\
\hline $1-3 \mathrm{yr}$ & $100(20.6)$ & 1794 (33.3) \\
\hline $4-6 \mathrm{yr}$ & $52(10.7)$ & $891(16.6)$ \\
\hline $7-13 \mathrm{yr}$ & $165(34.0)$ & $988(18.4)$ \\
\hline $14-21 \mathrm{yr}$ & $89(18.3)$ & $440(8.2)$ \\
\hline \multicolumn{3}{|l|}{ Birth year } \\
\hline 1992-1996 & $49(10.1)$ & $340(6.3)$ \\
\hline $1997-2000$ & $110(22.6)$ & $517(9.6)$ \\
\hline 2001-2004 & $93(19.1)$ & $569(10.6)$ \\
\hline $2005-2008$ & $75(15.4)$ & $1435(26.7)$ \\
\hline 2009-2012 & $159(32.7)$ & $2520(46.8)$ \\
\hline Sex, male & $235(48.4)$ & $2764(51.4)$ \\
\hline Neighbourhood income quintile & $n=478$ & $n=5314$ \\
\hline 1 (lowest) & $90(18.8)$ & $1121(21.1)$ \\
\hline 2 & $87(18.2)$ & $954(18.0)$ \\
\hline 3 & 95 (19.9) & $997(18.8)$ \\
\hline 4 & $87(18.2)$ & $1162(21.9)$ \\
\hline 5 (highest) & $119(24.9)$ & $1080(20.3)$ \\
\hline Rural residence & $\begin{array}{c}n=484 \\
116(23.9)\end{array}$ & $\begin{array}{l}n=5360 \\
869(16.2)\end{array}$ \\
\hline \multicolumn{3}{|l|}{ Year of test } \\
\hline 2009 & $<6(<1)$ & $82(1.5)$ \\
\hline 2010 & $35(7.2)$ & $1087(20.2)$ \\
\hline 2011 & $63(12.9)$ & $1083(20.1)$ \\
\hline 2012 & $366(75.3)$ & $2648(49.2)$ \\
\hline 2013 & $20(4.1)$ & $481(8.9)$ \\
\hline $\begin{array}{l}\text { Any complex chronic medical } \\
\text { condition }\end{array}$ & $9(1.9)$ & $262(4.9)$ \\
\hline \multicolumn{3}{|l|}{ Health care use in previous year } \\
\hline$\geq 2$ emergency department visits & $123(25.3)$ & $2070(38.5)$ \\
\hline$\geq 1$ hospital admission & 89 (18.3) & $1796(33.4)$ \\
\hline$\geq 8$ physician office visits & $140(28.8)$ & $2916(54.2)$ \\
\hline $\begin{array}{l}\text { Note: } \mathrm{SD}=\text { standard deviation. } \\
\text { *Unless stated otherwise. }\end{array}$ & & \\
\hline
\end{tabular}

those with up-to-date or partial vaccination and an adjusted OR of 1.21 (95\% CI 1.12 to 1.30 ) for those who received the DTaP vaccine only.

Compared with those who received at least 3 priming doses of whole-cell vaccine, individuals who received only acellular vaccine had an adjusted OR of 2.15 (95\% CI 1.30 to 3.57). Compared with participants who received 1 or more whole-cell vaccinations, those who received acellular vaccine for their priming series had an adjusted OR of 1.82 (95\% CI 1.18 to 2.82). The results from the sensitivity analysis evaluating participants who received a dose in 1997 assigned as either whole cell or acellular were similar (data not shown).

\section{Sensitivity analysis}

Accounting for nondifferential misclassification bias of vaccination status, vaccine effectiveness increased from $80 \%-85 \%$ to $95 \%$ or greater within 3 years since last vaccination. However, vaccine effectiveness was $63 \%$ after 4-7 years and declined to $22 \%$ (95\% CI $-70 \%$ to $73 \%$ ) after 8 or more years (Table 2, Appendix 2). Excluding infants with an incomplete primary series, vaccine effectiveness increased within 1 year from last vaccination from $80 \%$ to $87 \%$. Individuals tested in 2012-2013 were the main drivers of the waning immunity observed (Table 2).

\section{Interpretation}

We observed high effectiveness of the pertussis vaccine within 3 years of vaccination, but with clear evidence of waning of immunity beyond 4 years and little-to-no protection beyond 7 years from last vaccination. The odds of pertussis increased by $27 \%$ each year that passed after receipt of an acellular vaccine. Individuals primed with acellular vaccine had a 2.2 times higher odds of disease than those primed with the previously used whole-cell vaccine.

Recent observational studies from the US have yielded some comparable results. ${ }^{10,11,26-28}$ Our analysis, which is stratified by time since last vaccination and adjusted for age, suggests that the low estimates of vaccine effectiveness beyond 4 years from last vaccination are attributable to waning immunity. A recent metaanalysis identified an increased odds of pertussis of $33 \%$ for each year following the DTaP dose. ${ }^{23}$ That meta-analysis supports our finding that beyond 7 years since the last vaccination there is minimal protective effect of the vaccine, despite some evidence showing persistent humoural immunity. ${ }^{29}$ The findings of the current study are important because of its context. Our study population of Ontario residents 
received a 5-component acellular vaccine with consistently high coverage levels, had a history of a different whole-cell vaccine from the US and had no substantial population-wide resurgences of disease, yet our results showed similar rapid waning of immunity. Furthermore, our finding that the low-effectiveness whole-cell vaccine is still better for priming than the currently used acellular vaccine nearly 2 decades after the change in practice has profound implications for understanding the effectiveness of the pertussis vaccine.

Ontario transitioned to acellular vaccines owing to concerns about safety and effectiveness of the whole-cell vaccine used in Canada. Observational data from Canada, Australia and the US have shown greater protection with whole-cell infant priming. ${ }^{7-9}$ We were surprised to see a significant protective effect, given the estimated low effectiveness of this particular whole-cell vaccine of $49 \%-61 \% .^{3-5}$ However, our results showed that receiving 1 or more doses of whole-cell vaccine as an infant provided significant protection from pertussis more than a decade later.

Immunization advisory committees in Canada and the US recommend a Tdap booster for adolescents and adults. However, uptake of these recommendations as measured by pertussis vaccine coverage in Ontario and Canada has been about $70 \%$ among adolescents and only $7 \%$ among adults. ${ }^{30-33}$ Given our data, combined with reports from others, ${ }^{10,11}$ it is unsurprising that we are witnessing a resurgence of pertussis in adolescents and adults. ${ }^{2,11}$ The presence of a large susceptible population puts young infants, in whom virtually all of the morbidity and mortality is observed, at risk of acquiring infection. ${ }^{1}$

Our findings suggest the need to reconsider our strategy for pertussis vaccination. In Ontario, individuals currently aged 18 years and younger have received only acellular vaccines, creating a large susceptible population. Vaccination in pregnancy is recommended in the US and the United Kingdom, and is likely an effective strategy to reduce disease in infants. ${ }^{34}$ We recommend that decisions to strengthen control of pertussis be informed by dynamic modelling to compare the relative costs and benefits of different strategies. Consideration should also be given to introducing whole-cell vaccine for infant priming given the observational data showing significant long-term protective effects combined with the discovery that many cases of encephalopathy, temporally associated with the diphtheria and tetanus toxoids and pertussis (DTP) vaccine, were not causally attributable to pertussis vaccines. ${ }^{35,36} \mathrm{~A}$ recent model evaluating a 1-dose whole-cell priming strategy suggests that this approach is highly cost-effective. ${ }^{37}$ The benefit of additional boosters likely varies based on the cause of pertussis resurgence, which suggests that a better understanding of the current epidemiology of pertussis is critical for optimizing public policy. ${ }^{38}$

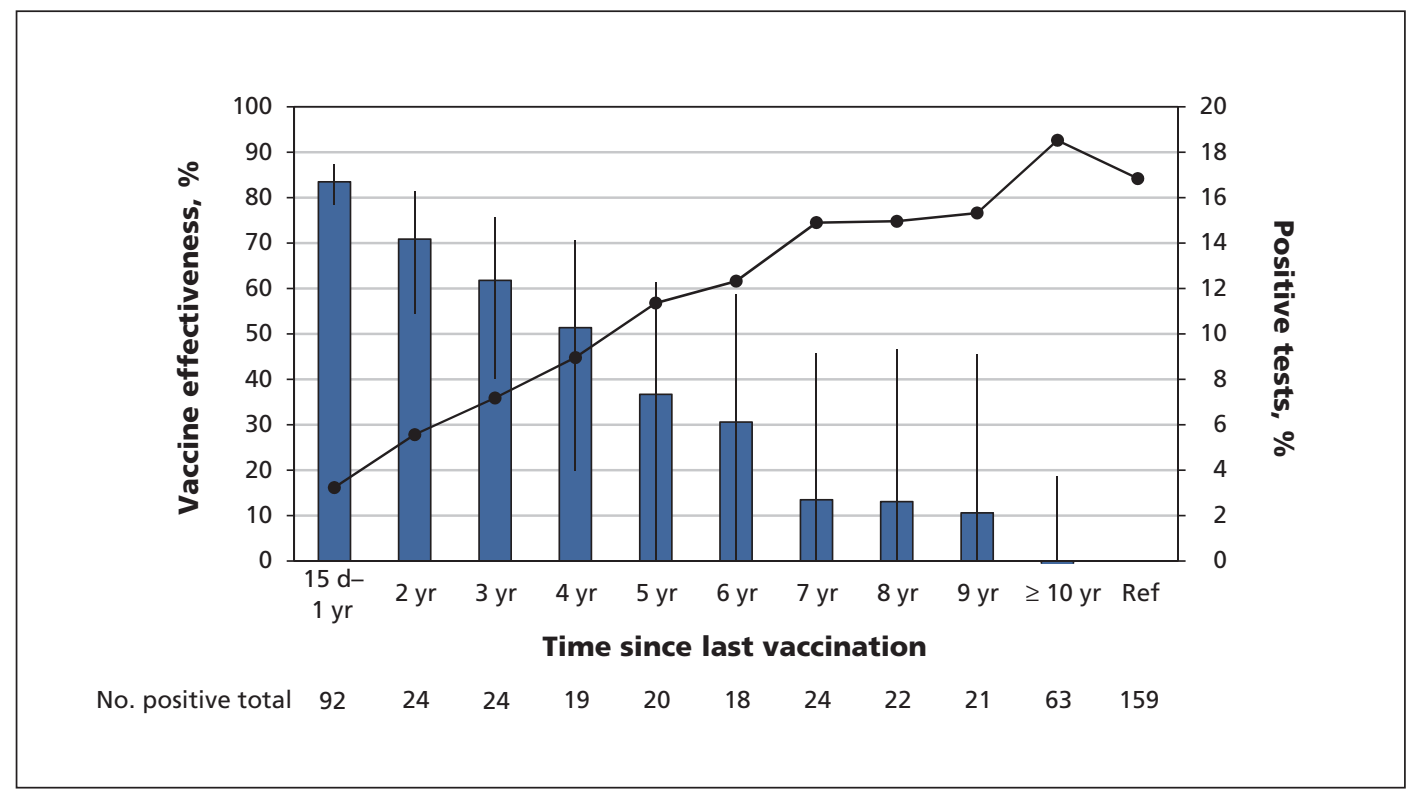

Figure 1: Pertussis-vaccine effectiveness (blue bars, left axis) and proportion of positive tests (solid line, right axis) by year since last vaccination in participants with up-to-date or partial vaccination. As time from last vaccination increased, vaccine effectiveness decreased, and the proportion of tests positive for pertussis increased. Vaccine effectiveness was calculated from crude odds ratios (ORs) using the formula VE = 1 OR $\times 100$. Error bars represent $95 \%$ confidence intervals. Ref $=$ reference group (unvaccinated population). Results of the Cochran-Armitage trend test for proportion of pertussis cases: $p<0.001$. 
Table 2: Crude and adjusted estimates of vaccine effectiveness, including sensitivity analyses*

\begin{tabular}{|c|c|c|c|c|}
\hline Variable; time since last vaccination & $\begin{array}{c}\text { Pertussis } \\
\text { positive, no. } \\
\text { vaccinated/total }\end{array}$ & $\begin{array}{c}\text { Pertussis } \\
\text { negative, no. } \\
\text { vaccinated/total }\end{array}$ & $\begin{array}{c}\text { Crude vaccine } \\
\text { effectiveness, \% } \\
(95 \% \mathrm{Cl})\end{array}$ & $\begin{array}{c}\text { Adjustedt } \\
\text { vaccine } \\
\text { effectiveness, \% } \\
(95 \% \mathrm{Cl})\end{array}$ \\
\hline \multicolumn{5}{|l|}{ Up-to-date vaccination } \\
\hline $15-364 d$ & $54 / 213$ & $1691 / 2476$ & 84 (78 to 89$) \S$ & 80 (71 to 86$) \S$ \\
\hline $1-3 \mathrm{yr}$ & $46 / 205$ & $1089 / 1874$ & 79 (71 to 85$) \S$ & 84 (77 to 89$) \S$ \\
\hline \multicolumn{5}{|l|}{ Partial vaccination } \\
\hline $15-364 \mathrm{~d}$ & $13 / 172$ & $340 / 1125$ & 81 (66 to 89$) \S$ & $75(54-86) \S$ \\
\hline $1-3 \mathrm{yr}$ & $27 / 186$ & $344 / 1129$ & 61 (41 to 75$) \S$ & $68(50$ to 80$) \S$ \\
\hline $4-7 \mathrm{yr}$ & $30 / 189$ & $223 / 1008$ & $34(-1$ to 56$)$ & 56 (30 to 72$) \S$ \\
\hline$\geq 8 \mathrm{yr}$ & $64 / 223$ & $305 / 1090$ & $-4(-43$ to 25$)$ & $36(-6$ to 61$)$ \\
\hline $1-3 \mathrm{yr}$ & $46 / 205$ & $1089 / 1874$ & $98(93$ to 100$) \S$ & $98(94$ to 100$) \S$ \\
\hline $4-7 \mathrm{yr}$ & $51 / 210$ & $391 / 1176$ & $56(27$ to 80$) \S$ & 63 (35 to 82$) \S$ \\
\hline$\geq 8 \mathrm{yr}$ & $42 / 201$ & $213 / 998$ & 14 (-61 to 67$)$ & 22 (-70 to 73$)$ \\
\hline \multicolumn{5}{|c|}{ Excluding infants aged $<7$ mo with incomplete primary series } \\
\hline $15-364 d$ & $26 / 158$ & $1192 / 1761$ & $91(86$ to 94$) \S$ & 87 (79 to 92$) \S$ \\
\hline $1-3 \mathrm{yr}$ & $46 / 178$ & $1089 / 1658$ & $82(74$ to 87$) \S$ & $84(77$ to 89$) \S$ \\
\hline $4-7 \mathrm{yr}$ & $51 / 183$ & $391 / 960$ & $44(20$ to 60$) \S$ & $62(42$ to 75$) \S$ \\
\hline$\geq 8 \mathrm{yr}$ & $42 / 174$ & $213 / 782$ & $15(-24$ to 42$)$ & $42(0$ to 66$)$ \\
\hline \multicolumn{5}{|c|}{ Indeterminate pertussis PCR considered positive } \\
\hline $15-364 d$ & $105 / 305$ & $1691 / 4276$ & $76(69$ to 81$) \S$ & $71(61$ to 79$) \S$ \\
\hline $1-3 \mathrm{yr}$ & $82 / 282$ & $1089 / 1874$ & 70 (61 to 78$) \S$ & 79 (72 to 85$) \S$ \\
\hline \multicolumn{5}{|l|}{ Restricted to years 2009-2011 } \\
\hline $15-364 \mathrm{~d}$ & $22 / 53$ & $839 / 1144$ & $74(55$ to 85$) \S$ & 71 (43 to 85$) \S$ \\
\hline $1-3 \mathrm{yr}$ & $13 / 44$ & $455 / 760$ & $72(45$ to 86$) \S$ & 84 (65 to 92$) \S \rrbracket$ \\
\hline $4-7 \mathrm{yr}$ & $11 / 42$ & $144 / 449$ & 25 (-54 to 63$)$ & 70 (26 to 87$) \S$ \\
\hline$\geq 8 \mathrm{yr}$ & $4 / 35$ & $61 / 366$ & 28 (-70 to 69$)$ & 79 (28 to 94$) \S \rrbracket$ \\
\hline \multicolumn{5}{|l|}{ Restricted to years 2012-2013 } \\
\hline $15-364 d$ & $32 / 160$ & $852 / 1332$ & $86(79$ to 91$) \S$ & 83 (73 to 89$) \S$ \\
\hline $1-3 \mathrm{yr}$ & $33 / 161$ & $634 / 1114$ & 81 (71 to 87$) \S$ & 85 (76 to 90$) \S \rrbracket$ \\
\hline $4-7 \mathrm{yr}$ & $40 / 168$ & $247 / 727$ & 39 (4 to 62$) \S$ & 57 (29 to 73$) \S$ \\
\hline$\geq 8 \mathrm{yr}$ & $38 / 166$ & $152 / 632$ & $6(-41$ to 38$)$ & 24 (-38 to 51)ף \\
\hline \multicolumn{5}{|l|}{ Excluding outbreak } \\
\hline $15-364 d$ & $48 / 139$ & $1578 / 2250$ & 78 (68 to 84$) \S$ & 76 (63 to 84$) \S$ \\
\hline $1-3 \mathrm{yr}$ & $38 / 129$ & $974 / 1646$ & $71(57$ to 81$) \S$ & $82(72$ to 88$) \S$ \\
\hline $4-7 \mathrm{yr}$ & $34 / 125$ & $342 / 1014$ & $27(-12$ to 52$) \S$ & 65 (41 to 79$) \S$ \\
\hline$\geq 8 \mathrm{yr}$ & $25 / 116$ & $183 / 855$ & -1 (-62 to 37$)$ & 55 (13 to 77$) \S ף$ \\
\hline \multicolumn{5}{|c|}{$\begin{array}{l}\text { Note: } \mathrm{Cl}=\text { confidence interval, } \mathrm{PCR}=\text { polymerase chain reaction. } \\
\text { *Reference group was the unvaccinated population. } \\
\text { †Adjusted for age, sex, rural v. urban residence, income quintile, any comorbidity, ambulatory health care use in previous } 12 \text { months, emergency health care use } \\
\text { in previous } 12 \text { months and inpatient health care use in previous } 12 \text { months. } \\
\text { fAdditional details in Appendix } 2 \text {, available at www.cmaj.ca/lookup/suppl/doi:10.1503/cmaj.160193/-/DC1. } \\
\text { \$p<0.05. } \\
\text { १The variable "any comorbidity" was excluded from these models owing to quasi-separation of data points. }\end{array}$} \\
\hline
\end{tabular}




\section{Limitations}

There is potential for false-negative results (cases being misclassified as controls) with the test-negative design, which would tend to underestimate vaccine effectiveness. However, test-positive cases are likely more severe than all pertussis cases in the population, ${ }^{39}$ thereby potentially overestimating vaccine effectiveness. This design minimizes the bias related to health care-seeking behaviour and has been validated against RCT data for other infections such as influenza and respiratory syncytial virus. ${ }^{14}$ In Ontario, pertussis vaccinations are administered predominately through physician offices. Nonetheless, some misclassification bias of vaccination status was observed. Our sensitivity analysis showed an underestimation of early vaccine effectiveness with a more dramatic waning of immunity over time. We incorporated all clinically relevant available covariates in the multivariable models to adjust for potential confounding; however, additional unmeasured confounders that could not be captured by administrative data are possible.

\section{Conclusion}

We identified good early vaccine effectiveness, which rapidly declined when time since last vaccination surpassed 4 years, in a population with relatively stable disease incidence. We also identified a significant protective effect from priming with a low-effectiveness whole-cell product. This study highlights the need to re-evaluate strategies for pertussis vaccination in Canada and to spur vaccine development.

\section{References}

1. Pertussis (whooping cough): for health care professionals Ottawa: Health Canada; modified 2014. Available: www.phac -aspc.gc.ca/im/vpd-mev/pertussis/professionals-professionnels -eng.php (accessed 2014 May 29).

2. Deeks SLLG, Walton R, Fediurek J, et al. Prolonged pertussis outbreak in Ontario originating in an under-immunized religious community. Can Commun Dis Rep 2014;40;42-9.

3. Bentsi-Enchill AD, Halperin SA, Scott J, et al. Estimates of the effectiveness of a whole-cell pertussis vaccine from an outbreak in an immunized population. Vaccine 1997;15:301-6.

4. De Serres G, Boulianne N, Duval B, et al. Effectiveness of a whole cell pertussis vaccine in child-care centers and schools. Pediatr Infect Dis J 1996;15:519-24.

5. Halperin SA, Bortolussi R, MacLean D, et al. Persistence of pertussis in an immunized population: results of the Nova Scotia Enhanced Pertussis Surveillance Program. J Pediatr 1989; 115:686-93.

6. Publicly Funded Immunization Schedules for Ontario - October 2015. Toronto: Ministry of Health and Long-Term Care; 2015. Available: www.health.gov.on.ca/en/pro/programs/immunization/ docs/immunization_schedule.pdf (accessed 2016 Sept. 12).

7. Vickers D, Ross AG, Mainar-Jaime RC, et al. Whole-cell and acellular pertussis vaccination programs and rates of pertussis among infants and young children. CMAJ 2006;175:1213-7.

8. Klein NP, Bartlett J, Fireman B, et al. Comparative effectiveness of acellular versus whole-cell pertussis vaccines in teenagers. Pediatrics $2013 ; 131: 1716-22$.

9. Sheridan SL, Ware RS, Grimwood K, et al. Number and order of whole cell pertussis vaccines in infancy and disease protection. JAMA 2012;308:454-6.
10. Klein NP, Bartlett J, Rowhani-Rahbar A, et al. Waning protection after fifth dose of acellular pertussis vaccine in children. N Engl J Med 2012;367:1012-9.

11. Baxter R, Bartlett J, Rowhani-Rahbar A, et al. Effectiveness of pertussis vaccines for adolescents and adults: case-control study. BMJ 2013;347:f4249.

12. Kwong JC, Campitelli MA, Gubbay JB, et al. Vaccine effectiveness against laboratory-confirmed influenza hospitalizations among elderly adults during the 2010-2011 season. Clin Infect Dis 2013;57:820-7.

13. Jackson ML, Nelson JC. The test-negative design for estimating influenza vaccine effectiveness. Vaccine 2013;31:2165-8.

14. De Serres G, Skowronski DM, Wu XW, et al. The test-negative design: validity, accuracy and precision of vaccine efficacy estimates compared to the gold standard of randomised placebocontrolled clinical trials. Euro Surveill 2013;18:pii: 20585.

15. Fisman DN, Tang $P$, Hauck $T$, et al. Pertussis resurgence in Toronto, Canada: a population-based study including testincidence feedback modeling. BMC Public Health 2011; 11:694.

16. Guthrie JL, Seah C, Brown S, et al. Use of Bordetella pertussi. BP3385 to establish a cutoff value for an IS481-targeted realtime PCR assay. J Clin Microbiol 2008;46:3798-9.

17. Guthrie JL, Robertson AV, Tang P, et al. Novel duplex realtime PCR assay detects Bordetella holmesii in specimens from patients with pertussis-like symptoms in Ontario, Canada. J Clin Microbiol 2010;48:1435-7.

18. Ducharme R, Benchimol EI, Deeks SL, et al. Validation of diagnostic codes for intussusception and quantification of childhood intussusception incidence in Ontario, Canada: a population-based study. J Pediatr 2013;163:1073-9.e3

19. Schwartz KL, Tu K, Wing L, et al. Validation of infant immunization billing codes in administrative data. Hum Vaccin Immunother 2015;11:1840-7.

20. Iron K, Zagorski BM, Sykora K, et al. Living and dying in Ontario: an opportunity for improved health information. ICES investigative report. Toronto: Institute for Clinical Evaluative Sciences; 2008.

21. Finkelstein MM. Ecologic proxies for household income: How well do they work for the analysis of health and health care utilization? Can J Public Health 2004;95:90-4.

22. Feudtner C, Christakis DA, Connell FA. Pediatric deaths attributable to complex chronic conditions: a population-based study of Washington State, 1980-1997. Pediatrics 2000;106: 205-9.

23. McGirr A, Fisman DN. Duration of pertussis immunity after DTaP immunization: a meta-analysis. Pediatrics 2015;135: 331-43.

24. Orenstein WA, Bernier RH, Dondero TJ, et al. Field evaluation of vaccine efficacy. Bull World Health Organ 1985;63:1055-68.

25. Fox MP, Lash TL, Greenland S. A method to automate probabilistic sensitivity analyses of misclassified binary variables. Int J Epidemiol 2005;34:1370-6.

26. Misegades LK, Winter K, Harriman K, et al. Association of childhood pertussis with receipt of 5 doses of pertussis vaccine by time since last vaccine dose, California, 2010. JAMA 2012; 308:2126-32.

27. Tartof SY, Lewis M, Kenyon C, et al. Waning immunity to pertussis following 5 doses of DTaP. Pediatrics 2013;131: e1047-52.

28. Liko J, Robison SG, Cieslak PR. Pertussis vaccine performance in an epidemic year - Oregon, 2012. Clin Infect Dis 2014;59:261-3.

29. Tomovici A, Barreto L, Zickler P, et al. Humoral immunity 10 years after booster immunization with an adolescent and adult formulation combined tetanus, diphtheria, and 5-component acellular pertussis vaccine. Vaccine 2012;30:2647-53.

30. Updated recommendations for use of tetanus toxoid, reduced diphtheria toxoid and acellular pertussis (Tdap) vaccine from the Advisory Committee on Immunization Practices, 2010. MMWR Morb Mortal Wkly Rep 2011;60:13-5.

31. Canadian Immunization Guide: Part 4, active vaccines: pertussis vaccine (February 2014). Ottawa: Public Health Agency of Canada; 2014. Available: www.phac-aspc.gc.ca/publicat/cig -gci/p04-pert-coqu-eng.php (accessed 2015 Mar. 1).

32. Immunization coverage report for school pupils: 2012-2013 Toronto: Public Health Ontario; 2014. Available: www.public healthontario.ca/en/eRepository/Immunization_coverage_report 2012-13.pdf (accessed 2016 Feb. 8)

33. Vaccine coverage amongst adult Canadians: results from the 2012 adult National Immunization Coverage (aNIC) survey. Ottawa: Public Health Agency of Canada; modified 2014 Available: www.phac-aspc.gc.ca/im/nics-enva/vcac-cvac-eng. php (accessed 2016 Feb. 8). 
34. Amirthalingam G, Andrews N, Campbell H, et al. Effectiveness of maternal pertussis vaccination in England: an observational study. Lancet 2014;384:1521-8.

35. Reyes IS, Hsieh DT, Laux LC, et al. Alleged cases of vaccine encephalopathy rediagnosed years later as Dravet syndrome. Pediatrics 2011;128:e699-702.

36. Berkovic SF, Harkin L, McMahon JM, et al. De-novo mutations of the sodium channel gene SCN1A in alleged vaccine encephalopathy: a retrospective study. Lancet Neurol 2006;5: 488-92.

37. DeAngelis H, Scarpino SV, Fitzpatrick MC, et al. Epidemiological and economic effects of priming with the whole-cell Bordetella pertussis vaccine. JAMA Pediatr 2016;170:459-65.

38. Riolo MA, Rohani P. Combating pertussis resurgence: one booster vaccination schedule does not fit all. Proc Natl Acad Sci U S A 2015;112:E472-7.

39. Bolotin S, Deeks SL, Marchand-Austin A, et al. Correlation of real time PCR cycle threshold cut-off with Bordetella pertussis clinical severity. PLoS One 2015;10:e133209.

Affiliations: Institute for Clinical Evaluative Sciences (Schwartz, Kwong, Campitelli, Stukel, Daneman); Institute of Health Policy, Management, and Evaluation (Schwartz, Stukel, Rosella, Daneman), University of Toronto; The Hospital for Sick Children (Schwartz); Public Health Ontario (Kwong, Deeks, Rosella, Rilkoff, Crowcroft); Dalla Lana School of Public Health (Kwong, Deeks, Bolotin, Crowcroft), University of Toronto; Department of Family and Community Medicine (Kwong), University of Toronto; University Health Network (Kwong); Department of Laboratory Medicine and Pathobiology (Jamieson, Marchand-Austin, Crowcroft), University of Toronto; Public Health Ontario
Laboratory Services (Jamieson, Marchand-Austin); Toronto, Ont.; ProvLab Alberta, University of Alberta Hospital (Drews); Department of Pathology and Laboratory Medicine (Drews), University of Alberta, Edmonton, Alta.

Contributors: All of the authors contributed to the conception, design, acquisition, analysis or interpretation of the data. Kevin Schwartz and Michael Campitelli performed the analysis, and Kevin Schwartz drafted the manuscript. All of the authors revised the manuscript, gave final approval of the version to be published and agreed to act as guarantors of the work.

Acknowledgement: The authors thank Cynthia Chen for her valuable data analysis.

Disclaimer: The opinions, results and conclusions reported in this paper are those of the authors and are independent from the funding sources. No endorsement by the Institute for Clinical Evaluative Sciences, Public Health Ontario or Ontario Ministry of Health and Long-Term Care is intended or should be inferred. Parts of this material are based on data and information compiled and provided by the Canadian Institute for Health Information (CIHI). However, the analyses, conclusions, opinions and statements expressed herein are those of the authors, and not necessarily those of CIHI.

Funding: This study was supported by the Canadian Institutes of Health Research, the Institute for Clinical Evaluative Sciences and Public Health Ontario, which are funded by annual grants from the Ontario Ministry of Health and LongTerm Care 